\title{
High-energy Few-cycle Pulses Directly Generated from Strongly Phase-mismatched Lithium Niobate Crystal
}

\author{
Zhou, Binbin; Chong, A.; Wise, F.W.; Bache, Morten
}

Published in:

CLEO Technical Digest

Publication date:

2012

Document Version

Publisher's PDF, also known as Version of record

Link back to DTU Orbit

Citation (APA):

Zhou, B., Chong, A., Wise, F. W., \& Bache, M. (2012). High-energy Few-cycle Pulses Directly Generated from Strongly Phase-mismatched Lithium Niobate Crystal. In CLEO Technical Digest (pp. QM4E.3). Optical Society of America.

\section{General rights}

Copyright and moral rights for the publications made accessible in the public portal are retained by the authors and/or other copyright owners and it is a condition of accessing publications that users recognise and abide by the legal requirements associated with these rights.

- Users may download and print one copy of any publication from the public portal for the purpose of private study or research.

- You may not further distribute the material or use it for any profit-making activity or commercial gain

- You may freely distribute the URL identifying the publication in the public portal 


\title{
High-energy Few-cycle Pulses Directly Generated from Strongly Phase-mismatched Lithium Niobate Crystal
}

\author{
B.B. Zhou ${ }^{1}$, A. Chong ${ }^{2}$, F. W. Wise ${ }^{2}$, M. Bache ${ }^{1}$ \\ ${ }^{1}$ DTU Fotonik, Technical University of Denmark, Bld. 343, DK-2800 Kgs. Lyngby, Denmark. \\ ${ }^{2}$ Department of Applied and Engineering Physics, Cornell University, Ithaca, New York 14853. \\ moba@fotonik.dtu.dk
}

\begin{abstract}
We show that effective soliton compression can be realized in strongly phasemismatched quadratic media. Sub-15 fs pulses are experimentally generated directly from 10 -mm-long bulk lithium niobate crystal by 120 -fs input pulses at $1300 \mathrm{~nm}$.

(C) 2011 Optical Society of America

OCIS codes: (320.5520) pulse compression; (320.7110) Ultrafast nonlinear optics; (190.5530) Pulse propagation and temporal solitons; (190.2620) Harmonic generation and mixing; (190.4400) Nonlinear optics, materials
\end{abstract}

\section{Introduction}

Energetic short-pulse laser sources are crucial for many research topics today and in the future, such as attosecond physics [1]. Due to the fact that high power lasers mostly give relatively long pulses, pulse compression techniques have been intensively investigated to break the limit. Among those techniques, noble-gas-filled hollow waveguide compressor and filamentation are mostly used; both rely on the cubic Kerr nonlinearity to broaden the spectrum. The self-focusing nature of cubic Kerr nonlinearity limits the pulse energy at a few millijoules level.

Another kind of compression approach uses self-defocusing nonlinearities based on phase-mismatched secondharmonic generation (SHG). A negative (self-defocusing) Kerr-like nonlinearity can be created during the frequency conversion and back-conversion process. It has several advantages. First, the self-focusing problems encountered in Kerr-based compressors are avoided, and therefore there is no bound to the input pulse energy except practical considerations such as the aperture size of the nonlinear crystal. Furthermore, both spectral broadening and temporal compression can occur in a single nonlinear material through solitons, since the negative nonlinearity could be balanced through normal material dispersion in the near-IR range [2].

To date, cascaded compressors have been heavily reported, but sub-20 fs results are rare [3]. The reason is due to the group velocity mismatch (GVM) between the fundamental and second-harmonic fields coupled in the nonlinear process. To get a strong negative cascaded nonlinearity so as to overcome the always existing positive cubic nonlinearity and maintain certain amount of effective negative nonlinearity, a low $\Delta k$ was typically chosen. For low $\Delta k$ values, the GVM makes the cascaded nonlinearity resonant and exclude short pulses generation [4]. This dilemma also restricts the usable nonlinear crystal to only few different kinds, such as $\beta$-barium borate (BBO) $[2,3,5]$ and periodically poled lithium niobate (PPLN) [6], all relying on type-I (birefringent) phase matching interaction.

We recently showed experimentally that effective soliton compression can be realized in strongly phase-mismatched quadratic media that relies on a different phase matching geometry, namely type 0 , where the fundamental and second harmonic waves have the same polarization. $50 \mathrm{fs}$ FWHM pulses at $1300 \mathrm{~nm}$ were compressed to $16 \mathrm{fs}$ in a $1 \mathrm{~mm}$ long LN crystal [7]. Here we demonstrate that strong compression can be achieved with longer input as well. With a piece of 10-mm-long bulk lithium niobate (LN) crystal, sub-15 fs pulses are directly generated with 120 fs input.

\section{Experiment}

Our experiment setup consists of a telescope system, a 10-mm-long, $5 \% \mathrm{MgO}$-doped X-cut congruent LN ( $\theta=\pi / 2$, $|\phi|=\pi / 2$, FW and SH polarized along the vertical optical Z-axis, beam propagating along X), some routing mirrors and a multiple-shot SHG FROG with a $30 \mu \mathrm{m}$ thick BBO crystal. The input pulses come from an optical parametric amplifier driven by a Ti:Sapphire regenerative amplifier. The pulse duration is measured to be $120 \mathrm{fs}$ with spectra FWHM width of $22 \mathrm{~nm}$. It is centered at $1300 \mathrm{~nm}$ and near transform-limited (time-bandwidth product $11 \%$ above the transform limit). The telescope system comprised by two curved silver mirrors is used to controlling the input beam spot size, and thus the input intensity. All the reflectors in the setup are silver mirrors. 




Fig. 1. The spectra of the direct input pulses and the pulses after propagating through the $\mathrm{LN}$ crystal under the optimum intensity

Best compression is observed under the input laser intensity of around $100 \mathrm{GW} / \mathrm{cm}^{2}$ (with the input pulse energy of $40 \mu \mathrm{J})$. Fig. 1 shows the input pulse spectrum and the typical output spectrum under the optimum intensity. The input spectrum component remains at large while two broad band secondary peaks appear around $1.2 \mu \mathrm{m}$ and $1.5 \mu \mathrm{m}$, respectively. The output spectrum could support pulses as short as 10.6 fs FWHM. The results of the FROG measurement with the output pulses are shown in Fig. 2. The retrieved temporal intensity shows clearly a main central spike and some small satellite pulses. The central spike gives 14.6 fs FWHM. The autocorrelation trace calculated from the FROG signal shows significant pedestal structure, caused by the satellite pulses. The retrieved FROG spectral intensity is in good agreement with the measured OSA spectrum in Fig. 1. Usually, in the initial compression stage a single soliton is formed with a compressed spike residing on a background (the pedestal), and only after further propagation does soliton fission into smaller constituents occur. This is not what we observe, but numerical simulations indicate that appearance of satellite pulses already at the initial compression stage could come from the material Raman effect [7]. We believe also by applying temperature control of the LN crystal and thus fine tune the $\chi^{(2)}$ coefficient, cleaner soliton and temporal pulse profile are possible. The dispersion in the directing beam path (from the exit face of the LN to the FROG) and inside the FROG itself also tends to exaggerate those satellite signals. To minimize that possible dispersion is also the future work for improvements.
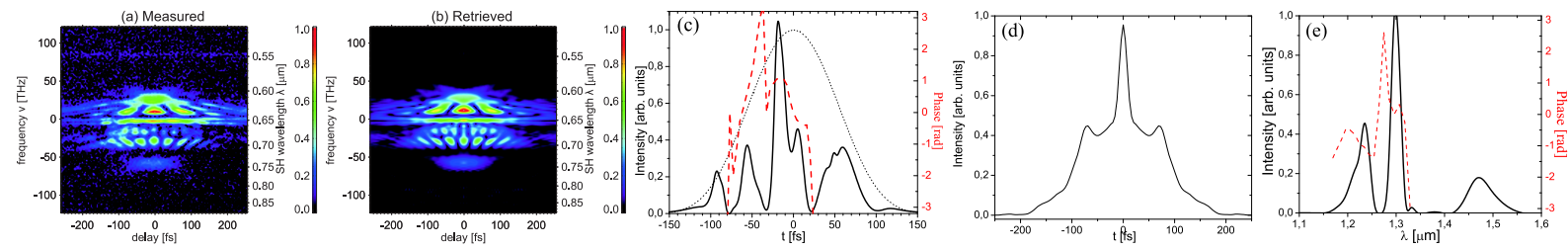

Fig. 2. FROG measurements at the optimum intensity. $(a, b)$ FROG traces (amplitude) of the output pulses. (c) The temporal amplitude and phase retrieved by the FROG algorithm, and the dot curve represents the input pulses. (d) The calculated FROG intensity autocorrelation trace. (e) The retrieved spectral amplitude and phase.

\section{Conclusion}

In conclusion, we show that bulk lithium niobate, the workhorse electro-optical material, also can be a very effective nonlinear medium for cascaded soliton compression. The potential to generate few-cycle pulses from a hundred fs level input has been experimentally demonstrated. $120 \mathrm{fs}$ transform-limited pulses at $1.3 \mu \mathrm{m}$ are compressed down to sub$15 \mathrm{fs}$ regime. The compression effect shown here is even better than by using PPLN structure, which has fabrication and aperture size limitations. It is also worth to mention that the technology in this abstract could be apply on the well developed high-energy Cr:forsterite laser system, the resulting few-cycle pulses could be the interesting drive source for high harmonic generation other than Ti:sapphire laser systems.

\section{References}

1. K. Krausz, and M. Ivanov, “Attosecond physics,” Rev. Mod. Phys. 81, 163 (2009)

2. X. Liu, L. Qian, and F. W. Wise, "High-energy pulse compression by use of negative phase shifts produced by the cascaded $\chi^{(2)}: \chi^{(2)}$ nonlinearity," Opt. Lett. 24, 1777-1779 (1999).

3. J. Moses and F. W. Wise, "Soliton compression in quadratic media: high-energy few-cycle pulses with a frequency-doubling crystal," Opt. Lett. 31, 1881-1883 (2006).

4. M. Bache, O. Bang, J. Moses, and F. Wise, "Nonlocal explanation of stationary and nonstationary regimes in cascaded soliton pulse compression," Opt. Lett. 32, 2490-2492 (2007).

5. J. Moses, E. Alhammali, J. M. Eichenholz, and F. W. Wise, "Efficient high-energy femtosecond pulse compression in quadratic media with flattop beams,"Opt. Lett. 32, 2469-2471 (2007).

6. S. Ashihara, T. Shimura, K. Kuroda, N. E. Yu, S. Kurimura, K. Kitamura, M. Cha, and T. Taira, "Optical pulse compression using cascaded quadratic nonlinearities in periodically poled lithium niobate,"App. Phy. Lett. 84, 1055-1057 (2004).

7. B.B. Zhou, A. Chong, F.W. Wise and M. Bache, "Few-cycle solitons in short strongly phase-mismatched frequency conversion crystals,"submitted, arXiv:1109.4261. 